\title{
Cryptogenic liver cirrhosis diagnosed in pregnancy and khat consumption
}

\author{
Tarek Khalife ${ }^{1}$, Gregory L. Goyert ${ }^{2}$, Roopina Sangha ${ }^{1}$, Ronald C. Strickler ${ }^{3}$ \\ ${ }^{1}$ Department of Obstetrics and Gynecology, Henry Ford Health System, Detroit, USA \\ ${ }^{2}$ Department of Obstetrics and Gynecology, Division of Maternal and Fetal Medicine, Henry Ford Health System, Detroit, USA \\ ${ }^{3}$ Departments of Obstetrics and Gynecology, Division of Reproductive Medicine, Henry Ford Health System, Detroit, USA \\ Email: tarekkhalife@hotmail.com
}

Received 2 October 2012; revised 4 November 2012; accepted 13 November 2012

\begin{abstract}
We report a case of cryptogenic liver cirrhosis likely due to khat consumption diagnosed in the setting of chronic hypertension and giving the appearance of atypical superimposed preeclampsia.
\end{abstract}

Keywords: Superimposed Preeclampsia; HELLP Syndrome; Liver Cirrhosis; Elevated Liver Enzymes; Khat

\section{INTRODUCTION}

Pregnancies complicated by chronic hypertension are associated with a substantial increase in the risk of superimposed preeclampsia, reaching as high as $25 \%$ [1,2]. Patients presenting with elevated liver enzymes in this setting will often be diagnosed to have atypical, superimposed preeclampsia (i.e. HELLP syndrome). Because of the variable presentation of patients with HELLP syndrome, and the significant maternal and neonatal morbidity associated with it, other clinical entities associated with elevated liver enzymes may not initially be considered in the differential diagnosis.

We present a case of a 37-year-old pregnant Yemeni patient who was found to have elevated liver enzymes with chronic hypertension and chronic mild thrombocytopenia. An initial diagnosis of atypical, superimposed preeclampsia guided the management; ultimately, however, early liver cirrhosis was diagnosed postpartum following an emergent cesarean section.

\section{CASE}

A 37-year-old G5 P4004 Yemeni patient at 30 weeks' gestation with chronic hypertension (no medication) and well-controlled gestational diabetes mellitus (GDM) Type A2 on glyburide, was admitted for mildly elevated liver enzymes $(A L T=59 \mathrm{IU} / \mathrm{L})$. Hepatic evaluation had previously been undertaken due to new-onset generalized pruritus at 27 weeks' gestation. The patient's obstetric history was uneventful with no previous history of preeclampsia OR GDM. Her prenatal course was unremarkable including normal aneuploidy screening results. Her medical history was pertinent for thyroidectomy on appropriate hormone replacement. The patient was born in Yemen and immigrated to the United States 14 years ago. She denied history of alcohol, cigarette or illicit drug consumption.

On presentation, the patient denied headaches, epigasric pain or visual symptoms. Her blood pressures ranged from 130 - 170/70 - $90 \mathrm{mmHg}$. Laboratory work up is listed in (Table 1).

The patient was admitted with a working diagnosis of chronic hypertension complicated by superimposed preeclampsia and possible HELLP syndrome. She received Betamethasone for acceleration of fetal lung maturity. The admission non-stress test was reactive and ultrasound documented a female fetus with an estimated weight of $1839 \mathrm{~g}$ (94th percentile), and an amniotic fluid index of $14 \mathrm{~cm}$.

The patient's blood pressure remained mildly elevated yet not requiring medications. Serial blood tests showed stable platelet counts and creatinine level, but her liver enzymes continued to rise slowly reaching a peak after one week of admission as demonstrated in (Table 1).

Her viral hepatitis profile, ANA screen and DNA

Table 1. Laboratory values at baseline, on admission and post partum in comparison to baseline 20 week values.

\begin{tabular}{|c|c|c|c|c|c|c|}
\hline & $\mathrm{Hb} g / \mathrm{dL}$ & Platelets/ $\mu \mathrm{L}$ & $\begin{array}{c}24 \mathrm{~h} \\
\text { Protein mg }\end{array}$ & $\begin{array}{l}\text { AST } \\
\text { IU/L }\end{array}$ & $\begin{array}{l}\text { ALT } \\
\text { IU/L }\end{array}$ & $\begin{array}{l}\text { Bilirubin } \\
\text { Total mg/dL }\end{array}$ \\
\hline Baseline & 11.2 & 105,000 & $320 \mathrm{mg}$ & 20 & 32 & 0.5 \\
\hline Admission & 10.6 & 83,000 & $330 \mathrm{mg}$ & 34 & 59 & 0.8 \\
\hline HD \#6 & 11.3 & 90,000 & $420 \mathrm{mg}$ & 49 & 116 & 0.6 \\
\hline Post Op & 8.0 & 107,000 & - & 25 & 23 & 0.3 \\
\hline
\end{tabular}


antibody were negative. Liver ultrasound was negative except for sludge in the gallbladder. The Hepatology service suggested an abdominal MRI to rule out portal hypertension and liver cirrhosis based on their experience with Yemeni patients, and also due to the presence of thrombocytopenia and mild hypoalbuminemia.

On the 8th hospital day, the patient experienced preterm premature rupture of membranes; parenteral antibiotics for prolongation of latency were started. The patient went into labor; an emergency cesarean section was done due to fetal mal-presentation. A female infant weighing $1847 \mathrm{~g}$ with Apgar scores of 3 at $1 \mathrm{~min}$ and 7 at 5 min was delivered; the placenta appeared normal and estimated blood loss was $600 \mathrm{~mL}$.

An abdominal MRI was obtained postpartum and showed evidence of early liver cirrhosis (Figure 1). Further postpartum maternal evaluation excluded hemochromatosis, $\alpha-1$ anti-trypsin deficiency, Wilson's disease and autoimmune hepatitis.

The postpartum course was uneventful except for asymptomatic anemia. The platelet counts remained mildly decreased and her liver enzymes normalized to an AST of $25 \mathrm{IU} / \mathrm{L}$ and ALT of $23 \mathrm{IU} / \mathrm{L}$. The patient was discharged home to follow up with the Hepatology service.

Two months after discharge, the patient experienced bleeding from ruptured esophageal varices and underwent endoscopic band ligation. Soon afterwards, she suffered from acute liver decompensation and was placed on the waiting list for liver transplantation.

\section{CONCLUSIONS}

Elevated liver enzymes and thrombocytopenia in pregnancy are frequently associated with adverse peri-natal outcomes [3]. The differential diagnosis includes severe preeclampsia, HELLP syndrome, thrombotic thrombocytopenic purpura, acute fatty liver, systemic lupus erythrematosis, cholestasis of pregnancy, and underlying

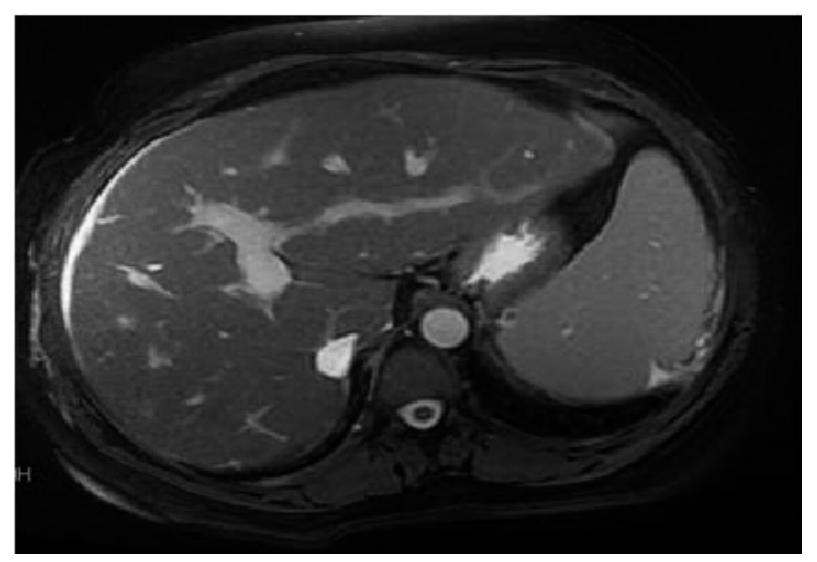

Figure 1. MRI of liver showing peri-portal fibrosis, portal hypertension, hepatosplenomegaly, a dilated portal vein of $17 \mathrm{~mm}$, and Cholilithiasis. hepatic dysfunction [4,5]. As with our patient, it can be very difficult to confirm an accurate diagnosis due to multiple confounding variables (i.e. chronic hypertension, proteinuria, chronic thrombocytopenia) [6]. The patient's Yemeni ethnicity prompted the Hepatologist to consider the potential of intrinsic hepatic pathology as has been observed in their practice with this ethnic group $[7,8]$. We hypothesize that the link could be environmental exposure to hepatotoxic substances related to cultural habits or practices (e.g. khat consumption) [9].

The consumption of Khat leaves (Catha edulis) is a widespread recreational custom in East Africa and the Arabian Peninsula. The plant contains the alkaloids cathine and cathinone, with amphetamine-like properties that produces a variety of pleasurable effects [10]. Khat is banned in the United States, but large quantities are nonetheless exported to North America from the United Kingdom, where it is available and used by the immigrant populations. The mechanism of injury is unknown but it is believed that ingestion of this plant induces recurrent episodes of subclinical autoimmune hepatitis that might eventually lead to cirrhosis. Another hypothesis is that the herbicides and fertilizers used to grow these plants could be the offending agent and not the plant itself [10].

We hypothesize that our patient's cryptogenic cirrhosis may have been related to the consumption of food products containing hepatotoxic substances used in Yemen, resulting in her very mixed clinical presentation. It is important for clinicians caring for pregnant patients belonging to certain ethnic backgrounds to be aware of this clinical entity; early referral and management may obviate some of the maternal morbidity attendant to hepatic decompensation [11]. Pregnancy outcomes with liver cirrhosis are favorable when the diagnosis is made early enough to avoid catastrophic outcomes [12,13]. Further studies focused on this ethnic group are needed to identify and confirm the offending agent.

\section{REFERENCES}

[1] Garitis, S., Sibai, B., Lindheimer, M., Hauth, J., et al. (1998) Risk factors for preeclampsia, abruptio placentae, and adverse neonatal outcomes among women with chronic hypertension. National Institute of Child Health and Human Development Network of Maternal-Fetal Medicine Units. The New England Journal of Medicine, 339, 667-671.

[2] Caritis, S., Sibai, B., Hauth, J., et al. (1998) Low dose aspirin to prevent preeclampsia in women at risk. National Institute of Child Health and Human Development Network of Maternal-Fetal Medicine Units. The New England Journal of Medicine, 228, 701-705. doi:10.1056/NEJM199803123381101

[3] Burrows, R.F. and Kelton, J.G. (1990) Thrombocytopenia 
at delivery: A prospective survey of 6715 deliveries. American Journal of Obstetrics \& Gynecology, 162, 731-734.

[4] Sibai, B.M. and Barton, J.R. (2007) Expectant management of severe Preeclampsia remote from term: Patient selection, treatment, and delivery indications. American Journal of Obstetrics \& Gynecology, 196, 514e1-514e9.

[5] Creasy, R. and Resnik, R. (2009) Diseases of the liver, biliary system, and pancreas. In: Williamson, C. and Mackillop, L. Eds., Maternal-fetal medicine. Principles and practice. 6th Edition, Saunders Elsevier, Philadelphia.

[6] Caldwell, S.H., Oelsner, D.H., Iezzoni, J.C., Hespenheide, E.E., Battle, E.H. and Driscoll, C.J. (1999) Cryptogenic cirrhosis: Clinical characterization and risk factors for underlying disease. Hepatology, 29, 664-669. doi:10.1002/hep.510290347

[7] Zheng, R.-Q., Huang, D.-M., Zhang, B., Su, Z.-Z., Kudo, M. and Kawasaki, T. (2005) Comparison of ultrasonography, CT and MRI in the diagnosis of liver cirrhosis. World Chinese Journal of Digestology, 13, 993-996.

[8] Alsubee, O. (2009) Internal medicine, Erfan Bagedo Hos- pital, Jeddah, Saudi Arabia: Khat and autoimmune heaptitis in men. The American Journal of Gastroenterology, 104, S117-S160.

[9] Neil, C. and Gezon, L. (2009) Khat in western Indian Ocean: Regional linkages and disjunctures. Etudes Ocean Indien, 42-43, 416.

[10] Chapman, M.H., Borges, O’Beirne, J., Crozier, A. and Crozier, A. (2010) Severe, acute liver injury and Khat leaves. The New England Journal of Medicine, 362, 1642-1644.

[11] Shaheen, A.A.M. and Myers, R.P. (2009) The outcomes of pregnancy in patients with cirrhosis: A populationbased study. Liver International, 30, 275-283.

[12] Mackillop, L. and Williamson, C. (2010) Liver disease in pregnancy. Postgraduate Medical Journal, 86, 160-164. doi:10.1136/pgmj.2009.089631

[13] Pajor, A. and Lehoczky, D. (1994) Pregnancy in liver cirrhosis-assessment of maternal and fetal risks in eleven patients and review of management. Gynecologic and Obstetric Investigation, 38, 45. doi:10.1159/000292444 